\title{
Rescue of prepulse inhibition deficit and brain mitochondrial dysfunction by pharmacological stimulation of the central serotonin receptor 7 in a mouse model of CDKL5 Deficiency Disorder
}

\author{
Daniele Vigli $^{\mathrm{a}}$, Laura Rusconi ${ }^{\mathrm{b}}$, Daniela Valentic ${ }^{\mathrm{c}}$, Paolo La Montanara ${ }^{\mathrm{b}}$, Livia Cosentino ${ }^{\mathrm{a}}$, Enza Lacivita ${ }^{\mathrm{d}}$, \\ Marcello Leopoldo ${ }^{\mathrm{d}}$, Elena Amendola ${ }^{\mathrm{e}}$, Cornelius Gross ${ }^{\mathrm{f}}$, Nicoletta Landsberger ${ }^{\mathrm{g}}$, Giovanni Laviola ${ }^{\mathrm{a}}$, \\ Charlotte Kilstrup-Nielsen ${ }^{\mathrm{b}}$, Rosa A. Vacca ${ }^{\mathrm{c}}$, Bianca De Filippis ${ }^{\mathrm{a}, *}$ \\ ${ }^{a}$ Center for Behavioral Sciences and Mental Health, Istituto Superiore di Sanità, 00161 Rome, Italy \\ b Department of Biotechnology and Life Sciences and Center of Neuroscience, University of Insubria, 21052 Busto Arsizio, Italy \\ ${ }^{c}$ Institute of Biomembranes, Bioenergetics and Molecular Biotechnologies, National Council of Research, 70126 Bari, Italy \\ d Dept. Pharmacy, University of Bari "Aldo Moro", 70125 Bari, Italy \\ e Molecular Medicine and Medical Biotechnologies, University of Naples "Federico II", 80131 Napoli, Italy \\ ${ }^{\mathrm{f}}$ Mouse Biology Unit, European Molecular Biology Laboratory (EMBL) 00015 Monterotondo, Italy \\ ${ }^{g}$ Department of Medical Biotechnology and Translational Medicine, University of Milan, 20090 Segrate, Italy
}

\begin{abstract}
A B S T R A C T
Mutations in the X-linked cyclin-dependent kinase-like 5 (CDKL5) gene cause CDKL5 Deficiency Disorder (CDD), a rare neurodevelopmental syndrome characterized by severe behavioural and physiological symptoms. No cure is available for CDD. CDKL5 is a kinase that is abundantly expressed in the brain and plays a critical role in neurodevelopmental processes, such as neuronal morphogenesis and plasticity. This study provides the first characterization of the neurobehavioural phenotype of 1 year old $C d k l 5$-null mice and demonstrates that stimulation of the serotonin receptor $7\left(5-\mathrm{HT}_{7} \mathrm{R}\right)$ with the agonist molecule LP-211 $(0.25 \mathrm{mg} / \mathrm{kg}$ once/day for 7 days $)$ partially rescues the abnormal phenotype and brain molecular alterations in Cdkl5-null male mice. In particular, LP-211 treatment completely normalizes the prepulse inhibition defects observed in Cdkl5-null mice and, at a molecular level, restores the abnormal cortical phosphorylation of rpS6, a downstream target of mTOR and S6 kinase, which plays a direct role in regulating protein synthesis. Moreover, we demonstrate for the first time that mitochondria show prominent functional abnormalities in Cdkl5-null mouse brains that can be restored by pharmacological stimulation of brain $5-\mathrm{HT}_{7} \mathrm{R}$.
\end{abstract}

\section{Introduction}

CDKL5 Deficiency Disorder (CDD) (OMIM \# 300672) is a rare neuropathological condition that is caused by mutations in the X-linked cyclin-dependent kinase-like 5 (CDKL5) gene (Kalscheuer et al., 2003). This disorder is characterized by a variety of behavioural and physiological symptoms that include the onset of seizures in the first months of life, severe global developmental delay resulting in intellectual disability (ID) and poor motor control, and the presence of peculiar hand stereotypies (Bahi-Buisson et al., 2008; Fehr et al., 2016). No cure exists for patients affected by CDD.
CDKL5 encodes a serine/threonine kinase expressed in various tissues, with the brain showing the highest levels of expression (Rusconi et al., 2008; Kilstrup-Nielsen et al., 2012). Available data point to a crucial role of Cdk15 in fundamental neurodevelopmental processes such as activity-dependent regulation of neuronal morphogenesis and plasticity (Fuchs et al., 2014; Zhou et al., 2017). These processes require a fine-tune regulation of Cdkl5 localization in neurons, with the shuttling between the cytoplasm and the nucleus being regulated by the activation of extra-synaptic NMDA receptors (Rusconi et al., 2011), and protein localization on the post-synaptic side of excitatory synapses being regulated by the association of the kinase with PSD-95 (Ricciardi et al., 2012). These neuronal alterations are accompanied by

\footnotetext{
* Corresponding author. Center for Behavioral Sciences and Mental Health, Istituto Superiore di Sanità, Viale Regina Elena, 299 , 00161 Roma, Italy.

Email address: bianca.defilippis@iss.it (B. De Filippis)
} 
a number of behavioural deficits in mice lacking Cdk15, including motor dysfunction, autistic-like behaviours and memory deficits (Jhang et al., 2017; Okuda et al., 2018).

The serotonin receptor $7\left(5-\mathrm{HT}_{7} \mathrm{R}\right)$ is a $\mathrm{G}$ protein-coupled receptor broadly expressed in the central nervous system that is involved in a variety of neurophysiological phenomena relevant for CDD, such as sleep, cognitive processes and synaptic plasticity (Hedlund et al., 2003; Cifariello et al., 2008; Matthys et al., 2011). Pharmacological stimulation of the $5-\mathrm{HT}_{7} \mathrm{R}$ by the brain penetrant agonist LP-211 has provided promising results in preclinical studies for disorders associated with syndromic IDs, such as Fragile X syndrome and Rett syndrome (RTT) (Costa et al., 2012; De Filippis et al., 2014a, 2015b). Of note, the beneficial effects of LP-211 treatment extend beyond intellectual deficits and impact other domains, such as motor function and autistic-like behaviours in a mouse model of RTT (De Filippis et al., 2014a, 2015b), a syndrome that presents several symptoms in common with CDD (Fehr et al., 2013). Moreover, $5-\mathrm{HT}_{7} \mathrm{R}$ stimulation by LP-211 impacts a number of behavioural domains and molecular pathways that have been demonstrated to be altered in Cdkl5-null mouse brain and in induced-pluripotent stem cell (iPSCs)-derived neurons from CDKL5 patients (Ricciardi et al., 2012; Amendola et al., 2014), as it promotes a rearrangement of neuronal morphology, facilitates synaptogenesis and modulates the activation of the Akt/mTOR/S6 pathway (De Filippis et al., 2014a; Speranza et al., 2017).

Based on these pieces of evidence, in the present study we evaluated whether the stimulation of $5-\mathrm{HT}_{7} \mathrm{R}$ might represent a potential therapeutic approach for CDD. To test this hypothesis, 9-12-months old Cdkl5-null male mice and wild-type littermate controls received a repeated systemic intraperitoneal (i.p.) treatment with LP-211 $(0.25 \mathrm{mg} /$ $\mathrm{kg}$ once/day for 7 days) (De Filippis et al., 2015b). We reasoned that the translational relevance of the treatment under investigation might be increased if the relief of symptoms was demonstrated at an advanced and more severely affected stage of the disease (1-year-old mice). Male mice were used based on clinical evidences of CDD in males (Elia et al., 2008) and on the effects of mosaic CDKL5 expression due to random X-chromosome inactivation in females. To evaluate therapeutic efficacy, a battery of behavioural analyses was carried out at the end of the treatment specifically tailored to detect CDD-related behavioural alterations. Behavioural testing started at least 7 days after the end of the i.p. treatment and the brains of the experimental mice were collected two months after the last i.p. injection, based on previous data suggesting long-term effects of a seven-day-long treatment with LP-211 (De Filippis et al., 2015b).

In the brain of Cdkl5-null mice treated with either LP-211 or vehicle, Rac1 activation and mitochondrial functionality were evaluated, since recent data suggest a role for $5-\mathrm{HT}_{7} \mathrm{R}$ in the activation of brain Rho GTPases and in the regulation of the oxidative phosphorylation (OXPHOS) apparatus, the mitochondrial molecular machinery responsible for the majority of cell energy production (De Filippis et al., 2015a, 2015b; Valenti et al., 2017), central players in several pathological conditions associated with ID (De Filippis et al., 2014b; Valenti et al., 2014). We also verified whether the expression and the activation of Rho GTPase-dependent pathways are abnormal in Cdkl5-null mouse brain and LP-211 treatment effects thereon, based on previous data pointing to a pathogenic role of a disrupted interaction between Cdkl5 and the Rho GTPases Rac1 (Chen et al., 2010; Barbiero et al., 2017). In particular, we explored whether the LP-211 treatment impacts group I PAKs, the leading molecules by which Rho GTPases affect actin cytoskeleton dynamics (De Filippis et al., 2014b), and the activation of the rpS6 and its upstream regulator Akt, a pathway that is modulated by Rho GTPases and is involved in protein synthesis (Ricciardi et al., 2011; De Filippis et al., 2014a).

\section{Materials and methods}

\subsection{Subjects}

The experimental subjects were 9-12-months old Cdkl5-null male mice and wild-type littermates (wt) backcrossed to C57BL/6N mice for at least 10 generations (Amendola et al., 2014). Experimental mice were obtained by crossing Cdkl5 heterozygous $(-/+)$ female mice and wt male mice and weaned at postnatal day 25 . After weaning, mice were housed according to sex in groups of two or three in polycarbonate transparent cages $(33 \times 13 \times 14 \mathrm{~cm})$ with sawdust bedding and kept on a 12-h light-dark schedule (lights off at 8:00 am). Temperature was maintained at $21 \pm 1{ }^{\circ} \mathrm{C}$ and relative humidity at $60 \pm 10 \%$. Animals were provided ad libitum with tap water and a complete pellet diet (Altromin, 1324 - $10 \mathrm{~mm}$ pellets, Germany). All experimental procedures were conducted in conformity with the European Directive 2010/63/EU and the Italian legislation on animal experimentation, D.Lgs. 26/2014.

\subsection{Genotyping}

DNA has been prepared from a small tail-tip biopsy taken at weaning, as previously described (De Filippis et al., 2014a). The Cdkl5 alleles have been identified by PCR using two sets of primers (for further details see Supplementary materials). PCR products were electrophoresed through a 2\% NuSieve 3:1 agarose gel (Cambrex Bio Science, Rockland, ME, USA) containing $0.1 \mu \mathrm{l} / \mathrm{ml}$ GelRed $^{\mathrm{TM}}$ and examined under UV light.

\subsection{Drug and treatment}

LP-211 was prepared following the same synthetic procedure described in (Leopoldo et al., 2008). The compound, which has a half-life $65 \mathrm{~min}$, was dissolved in a vehicle solution of $1 \%$ dimethyl sulfoxide (DMSO) in saline $(0.9 \% \mathrm{NaCl})$. Cdkl5-null mice and wt littermate controls were randomly assigned to be daily i.p. injected (between 9.00 and 11.00 a.m.) for 7 consecutive days with either LP-211 $(0.25 \mathrm{mg} / \mathrm{kg})$ or vehicle ( $1 \%$ of DMSO in saline).

\subsection{Behavioural testing}

A comprehensive test battery was carried out aimed at assessing treatment effects on the behavioural domains that are compromised in CDD. The selection of the tests to be performed was based on previous literature addressing the neurobehavioural phenotype of Cdkl5-null mice (Amendola et al., 2014; Okuda et al., 2018) and on our lasting experience on the study of mouse models of RTT, a syndrome that has many symptoms in common with CDD (De Filippis et al., 2010, 2015a). Mice were experimentally naïve at the start of the behavioural test battery. All behavioural testing took place during the dark phase of the L/ D cycle, between 9.00 a.m. and 3.00 p.m., and was carried out by experimenters blind to the mouse genotypes. A minimum of $24 \mathrm{~h}$ was left between tests.

\subsubsection{Prepulse inhibition (PPI) paradigm}

Sensorimotor gating was evaluated 7 days after the last i.p. with the prepulse inhibition (PPI) paradigm (Swerdlow et al., 2001). The apparatus consisted of two Plexiglas rectangular boxes (startle cages) $(9 \times 7 \mathrm{~cm})$, placed in sound-attenuated chambers with a red light and a fan ventilator (Med associates inc. St Albans, VT, United States of America). Background white $(62 \mathrm{db})$ noise and acoustic bursts were conveyed by two separate speakers, properly spaced from the startle cage so as to produce a fine-tuned regulation of sound. Both speakers 
and startle cages were connected to a main PC computer, which detected and analyzed all chamber variables by means of a specific software. Two slightly different protocols were adopted on two cohorts of mice, that differed in the range of prepulse intensities under investigation. On the first cohort of mice, prepulse intensities were as follows: $67,70,73$ or $76 \mathrm{db}$ (Macri et al., 2015). On the second cohort, 78, 82 or $84 \mathrm{~dB}$ pre-pulse intensities were applied (Chao et al., 2010). To evaluate sensorimotor gating capabilities in Cdkl5-null mice, the \% PPI was calculated as follows: (100-[(mean startle amplitude for prepulse + pulse trials/mean startle amplitude for pulse-alone trials) x 100]) (for further details see Supplementary materials).

\subsubsection{General health score}

The general health of the experimental mice was qualitatively evaluated 1 and 28 days after the last injection, by a trained observer, blind to the genotype of the experimental mice, according to a method that has been developed to assess the health status of RTT mice (Guy et al., 2007; De Filippis et al., 2014a). Briefly, mice received a score (ranging from 0 - normal appearance-to 4- highly compromised) for each of the following parameters: gait, mobility, breathing, kyphosis, fur, hind limb clasping, tremors and general conditions. The individual scores for each category were subsequently averaged to obtain a semi-quantitative measure of the general health status.

\subsubsection{Nest building evaluation}

Nest building ability was scored 21 days from the last i.p. injection to assess purposeful and coordinated forepaw use to unravel whether Cdkl5-null mice display alterations and LP-211 effects thereon, as previously described (De Filippis et al., 2015a). The quality of the nests was evaluated $24 \mathrm{~h}$ after nest material provision (for further details see Supplementary materials).

\subsubsection{Home cage locomotor activity}

To verify whether LP-211 treatment affects the daily locomotor activity in Cdkl5-null mice, spontaneous locomotor activity in the home-cages was evaluated 33 days after the last i.p. injection. Levels of activity were monitored continuously by means of an automatic device using small passive infrared sensors positioned on the top of each cage (ACTIVISCOPE system, NewBehaviour Inc., Zurich, Switzerland) as previously described (De Filippis et al., 2013) (for further details see Supplementary materials). To avoid confounding effects due to cage clean procedures and/or room entrances, the analysis was performed during two 6-h intervals, during the dark and the light phase, in which animals were left undisturbed.

\subsubsection{Open field test}

Locomotor activity was assessed in the Open Field test 30 days after the last i.p injection, to complement the home-cage recording (Ricceri et al., 2011) (for further details see Supplementary materials).

\subsubsection{Fear conditioning task}

The fear conditioning task was carried out 14 days after the last i.p. injection to evaluate cognitive abilities in Cdkl5-null mice and LP-211 effect thereon (Wang et al., 2012). An automated system was used (UgoBasile S.R.L.), which consisted in a soundproof cubic apparatus with inside a mouse cage (21(d) x $24(\mathrm{w})$ x $30(\mathrm{~h}) \mathrm{cm}$ ) with electrified grid floor. The task consisted of a two-days-long protocol in which freezing frequency and duration were measured with an automatic freezing detector (UgoBasile S.R.L.). Throughout the task, mice were exposed to a white noise $(\mathrm{WN}-60 \mathrm{db}, 2000 \mathrm{~Hz})$. On the first day (training), animals were placed in the fear conditioning apparatus for $180 \mathrm{~s}$ (baseline, BL) and then exposed for three times to the acoustic conditioned stimulus (CS; $2000 \mathrm{~Hz}-68 \mathrm{db}, 30 \mathrm{~s})$. Each CS on the first day was paired with a $0.7 \mathrm{~mA}$ shock released during the last $2 \mathrm{~s}$ (unconditioned stimulus; US). A 95s inter trial interval (ITI) was used. On the second day(test), mice were placed in the same chamber and, after a 180s BL, were exposed to fifteen trials consisting in 30s of CS plus 10s of ITI. Contextual fear memory was established by measuring the time spent in freezing behaviour during the baseline on the testing day compared to levels shown during the baseline on the training day. Freezing behaviour in response to the CSs on the test day was also evaluated. Before the starting of each session the grid floor of the apparatus was cleaned with $70 \%$ ethanol.

\subsection{Neurobiological analyses}

Two months after the last i.p. injection, the brains of the experimental mice were dissected and cortices, a behaviourally relevant brain area in which 5- $\mathrm{HT}_{7} \mathrm{R}$ (Hedlund, 2009) and Cdkl5 (Wang et al., 2012) are highly expressed, were immediately frozen in dry ice for G-LISA Assay and western blot analyses (De Filippis et al., 2015b).

For mitochondrial analyses, the hemispheres from additional subjects were cryopreserved, as previously described (Valenti et al., 2017). Previous data in fact demonstrate that cryopreserved brain tissues show mitochondrial membrane potential, outer and inner membrane integrity and mitochondrial ATP production capacity comparable to mitochondria isolated from fresh brains (Valenti et al., 2014).

\subsubsection{RAC-1 G-LISA assay}

Rac1 G-Lisa Activation Assay BiochemkitTM (Cytoskeleton, Denver, $\mathrm{CO})(\mathrm{n}=4-5)$ was used to measure Rac1 activity in mouse cortices according to the manufacturer's recommendations.

\subsubsection{Western blot analysis}

Cortices were homogenized in lysis buffer and centrifuged. Then the supernatant was collected and the protein content was quantified by bicinchonic acid assay. For western blotting analysis, $20 \mu \mathrm{g}$ of total proteins were separated on a $12 \%$ SDS-PAGE and membranes incubated with the appropriate primary and secondary antibodies. Images of the membranes were acquired by a CCD camera (Syngene, G-Box Chemi XRQ) and optical densities (O.D.) of the protein signals calculated for each sample with Image J software and normalized with the corresponding housekeeping signal (Fig. $4 \mathrm{~A}, \mathrm{C}$ ); the O.D. ratios were then compared and expressed as the average fold increase, with 1 (wt control) as baseline (for further details see Supplementary materials).

\subsubsection{Mitochondrial analysis}

Measurement of mitochondrial respiratory chain complex (MRC) activities. MRC activities were evaluated in mitochondrial membrane-enriched fractions obtained from isolated mitochondria. Measurement of MRC complex activities were performed essentially as in (Manente et al., 2013), by three assays which rely on the sequential addition of reagents to measure the activities of: i) NADH: ubiquinone oxidoreductase (complex I) followed by ATP synthase (complex V), ii) succinate: ubiquinone oxidoreductase (complex II) and iii) cytochrome c oxidase (complex IV) followed by cytochrome c oxidoreductase (complex III) (for further details see Supplementary materials).

Measurement of mitochondrial ATP production rate. The rate of ATP production by OXPHOS was determined in isolated mitochondria, as previously described in (Valenti et al., 2010) (for further details see Supplementary materials).

Measurement of mouse brain ATP levels. Half brain was weighted (approx. $20 \mathrm{mg}$ ) and subjected to perchloric acid extraction as described in (Khan, 2003) (for further details see Supplementary materials). The amount of tissue ATP was determined enzymatically in $\mathrm{KOH}$ neutralized extracts, as described in (Valenti et al., 2010). 


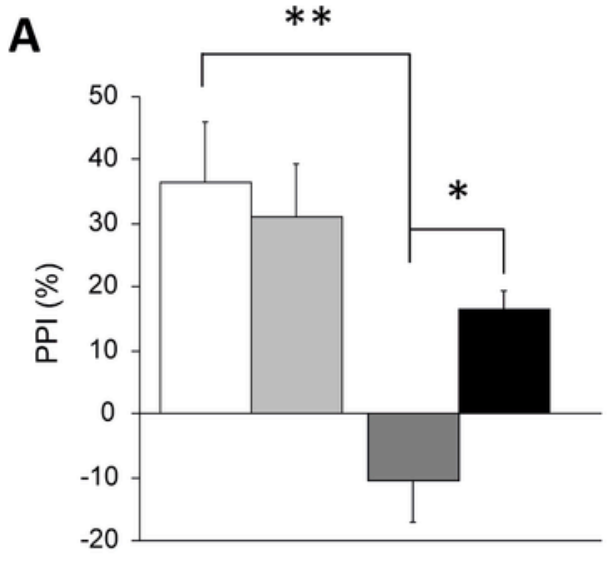

First cohort

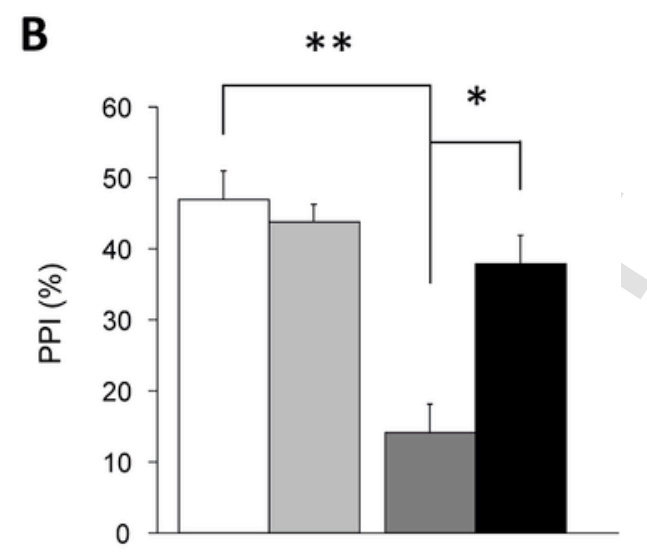

Second cohort

\section{wt, veh $\square$ wt, LP-211 $\square$ Cdkl5-null, veh $\square$ Cdkl5-null, LP-211}

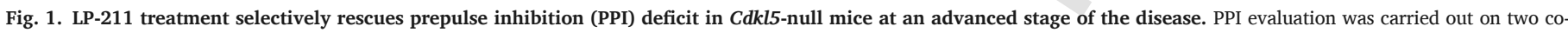

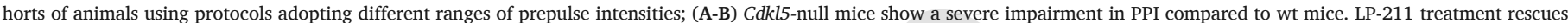

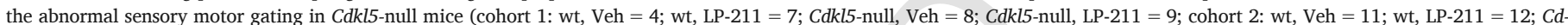

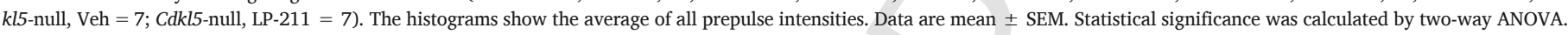
$* * \mathrm{p}<0,01 ; * \mathrm{p}<0,05$ after Tukey's post-hoc tests.

\subsection{Statistical analysis}

Data were analyzed using the ANOVA model, including genotype and treatment as between-subjects factors, or applying repeated measures ANOVAs if there was a within-subjects factor, using Statview vers. 5.0 (Sas, Institute Inc., Cary, NC). The alpha level was set to $5 \%$. To unravel the presence of outliers, the Grubbs' test was applied. Post-hoc comparisons were performed using Tukey HSD (Wilcox, 1987).

\section{Results}

3.1. LP-211 treatment selectively rescues PPI deficit in mice lacking Cdkl5 at an advanced stage of the disease

To evaluate the efficacy of the LP-211 treatment for CDD, a broad test battery was carried out.

Prepulse inhibition (PPI). The evaluation of the sensorimotor gating showed significant deficits in PPI capacity in Cdkl5-null mice compared to wild-type (wt) controls, in the absence of changes in the acoustic startle response (see Fig. S1 A-B). This genotype effect was replicated on two cohorts of animals using protocols adopting different ranges of prepulse intensities [Fig. 1A cohort 1: Genotype*Treatment interaction: $\mathrm{F}(1,22)=12.2, \mathrm{p}=0.021$; post-hoc: $\mathrm{p}<0.01$; Fig. $1 \mathrm{~B}$ cohort 2 : Genotype*Treatment interaction: $\mathrm{F}(1,33)=7.3, \mathrm{p}=0.011$; post-hoc: $\mathrm{p}<0.01]$.The LP-211 treatment significantly improved this abnormal behaviour in Cdkl5-null mice compared to vehicle (veh)-treated $C d$ kl5-null mice in both cohorts of animals [Fig. 1A, Genotype*Treatment; post-hoc: $\mathrm{p}<0.01$; Fig. 1B; Genotype*Treatment; post-hoc: $\mathrm{p}<0.05]$ ] No significant prepulses intensities*genotype*treatment interactions were found (Fig. S1 C-D).

General health status. We found that fully symptomatic Cdkl5-null mice showed worse general health conditions in comparison to wt mice [Fig. 2A; Genotype: F $(1,35)=7.8 ; \mathrm{p}=0.008$ ]. The LP-211 treatment did not significantly improve general health status in Cdkl5-null mice (Fig. 2A). No differences between the first and the second evaluation (1 and 28 days from the last i.p.), and no interaction of the repeated measures with genotype and treatment were found. Fig. $2 \mathrm{~A}$ represents the genotype*treatment interaction, in which the general health scores obtained at 1 and 28 days after the last i.p. injections were averaged.

Nest building ability. Nest building ability was slightly, but significantly impaired in Cdkl5-null mice in comparison to wt controls [Genotype: $\mathrm{F}(1,35)=4.9 ; \mathrm{p}=0.032$ ], thus confirming defective coordination of forepaws (De Filippis et al., 2015a; Fuchs et al., 2018b). The LP-211 treatment did not affect the quality of the nests built by Cdkl5-null mice (wt veh: $2.3 \pm 1.2$; Cdkl5-null veh: $1.8 \pm 1.4$; wt LP-211: $2.5 \pm 1.2$; Cdkl5-null LP-211: $1.3 \pm 1.0$ ).

Home cage locomotor activity. The evaluation of spontaneous home cage locomotor activity highlighted a hypoactive profile in Cdkl5-null mice compared to wt controls, as demonstrated by the lower number of beam breaks they performed during the dark/active phase of the Light/Dark cycle [Fig. 2B; Phase*Genotype*Treatment interaction: F $(1,28)=3.3 ; \mathrm{p}=0.082 ;$ post-hoc: $\mathrm{p}<0.05]$. The LP-211 treatment did not affect the abnormal locomotor profile shown by Cdkl5-null mice in the home cage.

Open field test. We found that Cdkl5-null mice show hyperactivity when exposed to a novel environment compared to wt controls, as demonstrated by the increased distance they moved in the open field [Fig. 2C; Genotype: $\mathrm{F}(1,33)=13.6 ; \mathrm{p}<0.001$ ] as well as the number of entrances in the central zone of the arena [Genotype: $F(1,33)=5.9$, $\mathrm{p}<0.021$ ]. Increased locomotion was confirmed throughout the 60 -min Open Field test, with no differences between the initial and the last 5-min blocks (Fig. S2). LP-211 treatment did not exert any effects on the total distance moved (Fig. 2C) as well as the number of entrances in the central zone of the arena (wt veh:191.5 \pm 65.1; Cdkl5-null mice veh: $222.4 \pm 83.9$; wt LP-211: $176.6 \pm 55.2$; Cdkl5-null mice LP-211: $252.6 \pm 60.7)$. No difference between Cdkl5-null mice and wt controls was found in time spent in the central/intimidating zone of the arena, an index of anxiety-like behaviours (data not shown).

Fear conditioning test. Defective contextual fear memory was found in Cdkl5-null mice, as demonstrated by the reduced freezing levels they displayed compared to wt controls when exposed to the context in which they received the footshock on the previous day [Fig. 2D; Day*Genotype*Treatment interaction: $\mathrm{F}(1,35)=3.1 ; \mathrm{p}=0.086$; post-hoc: $\mathrm{p}<0.01$ ]; no significant LP-211 treatment effect was highlighted on this hippocampus-dependent cognitive deficit. Reduced freezing 
A

Genotype: $p=0.008$

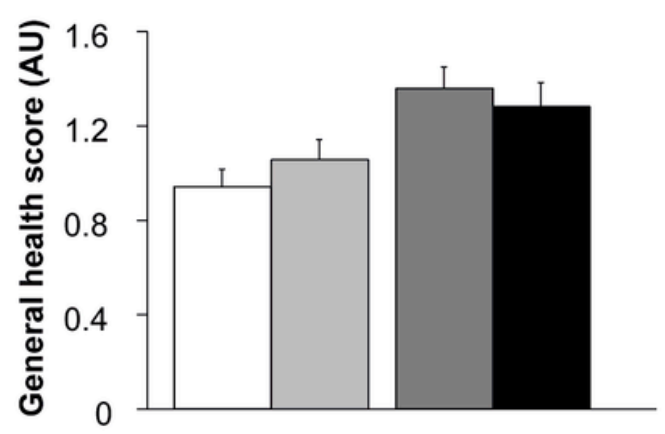

C

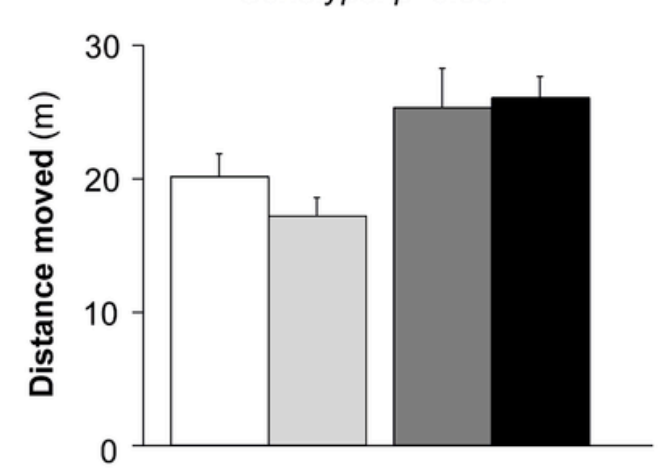

B

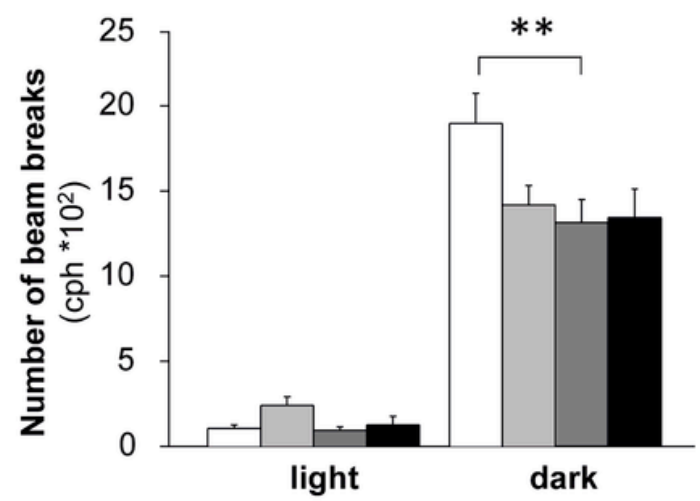

D

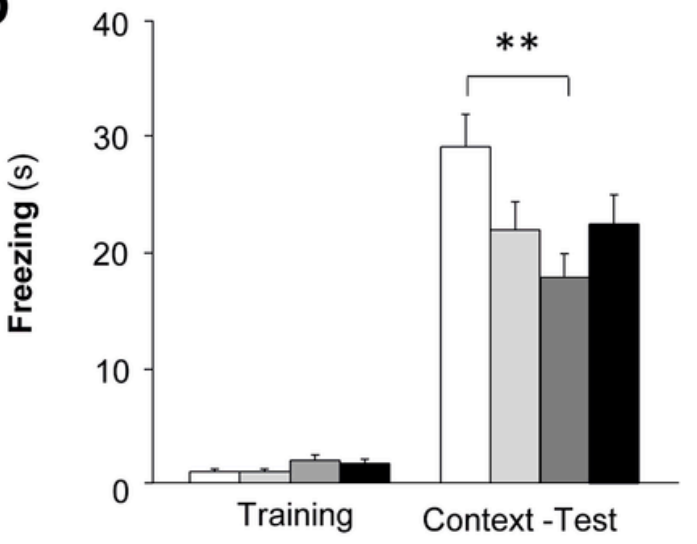

wt, veh

wt, LP-211

Cdk/5-null, veh

Cdk/5-null, LP-211

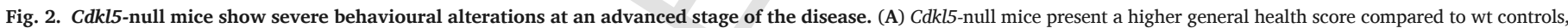

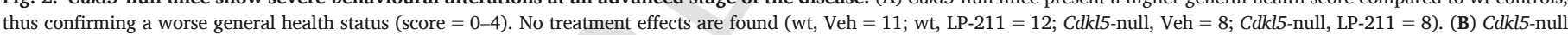

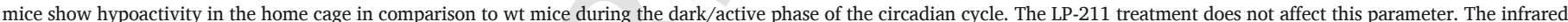

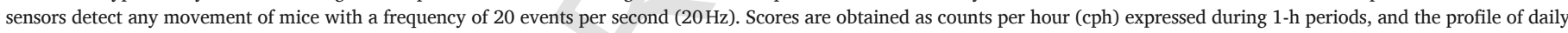

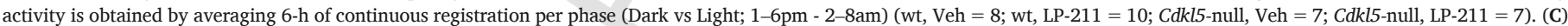

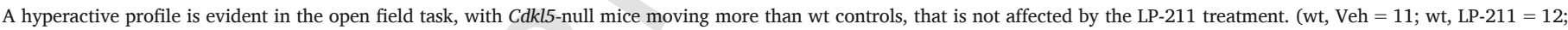

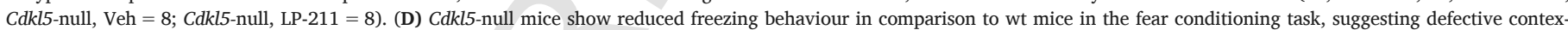

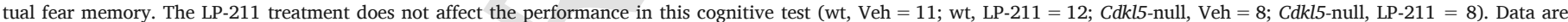
mean \pm SEM. Statistical significance was calculated by two-way ANOVA. **p $<0,01 ;{ }^{*} \mathrm{p}<0,05$ after Tukey'spost-hoc tests.

response to the presentation of the 15 CSs on the second day of testing compared to wt controls was also evident in Cdkl5-null mice [Genotype: $\mathrm{F}(1,35)=11.6 ; \mathrm{p}<0.001]$. The LP-211 treatment did not improve this abnormal freezing response shown by Cdkl5-null mice (wt veh:27.9 \pm 10.2 ; Cdkl5-null mice veh: $16.3 \pm 12.0$; wt LP-211: $24.1 \pm 11.8$; Cdkl5-null mice LP-211: $16.5 \pm 12.8$ ).

\subsection{The LP-211 treatment activates Rac1 and rescues the abnormal activation of rpS6 in the cortex of Cdkl5-null mice}

Based on available data suggesting that Rac1 signaling may be defective in CDD (Chen et al., 2010), the activation of Rac1 and of the Rho GTPases downstream molecules PAKs and rpS6 was evaluated in Cd$k l 5$-null mouse cortex, to verify whether they are abnormal and whether pharmacological stimulation of the $5-\mathrm{HT}_{7} \mathrm{R}$ may recover them.

Rac1 activation. No genotype difference was found in the activation of Rac1 in Cdkl5-null mouse cortex. The LP-211 treatment significantly increased Rac1 activation in both genotypes [Fig. 3, Treatment: F $(1,15)=5.8 ; \mathrm{p}=0.028]$.
Expression and activation of RhoGTPase-dependent signaling pathways. We found that phospho-PAK(p-PAK)/total PAK ratio, which provides an index of the net functionality of the kinase, was shifted toward increased activation in Cdkl5-null mouse cortex compared to wt controls [Fig. 4A and B; Genotype: F $(1,17)=15.7$; $\mathrm{p}<0.001$ ]. The LP-211 treatment increased PAK activation in the cortex of both genotypes, as demonstrated by increased p-PAK/total PAK ratio [Fig. 4B, Treatment: $\mathrm{F}(1,17)=11.7 ; \mathrm{p}=0.003]$.

In Cdkl5-null mouse cortex, we also observed increased ribosomal protein S6 (rpS6) activation (Fig. 4C representative blots), as demonstrated by increased phospho-rpS6 (240/244) (p-rpS6)/total rpS6 ratio, which was normalized by the LP-211 treatment [Fig. 4D; Genotype*Treatment interaction: $\mathrm{F}(1,18)=3.2, \mathrm{p}=0.089 ;$ post-hoc: $\mathrm{p}<0.05]$. No genotype or treatment effects were found on the phosphorylation levels of the rpS6 at Ser235/236 in the cortex (Fig. 4E).

Akt activation levels. In the cortex of Cdkl5-null mice, no genotype difference and no LP-211 treatment was detected for the activation of Akt quantified as the ratio phospho-Akt (p-Akt)/Akt total (Fig. S3). 
Treatment: $p=0.028$

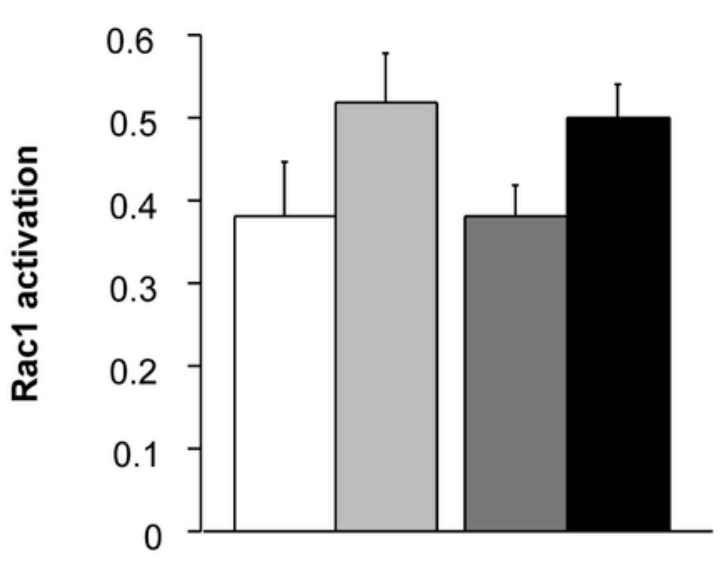

\section{wt, veh $\square$ wt, LP-211 $\square$ Cdk/5-null, veh $\square$ Cdk/5-null, LP-211}

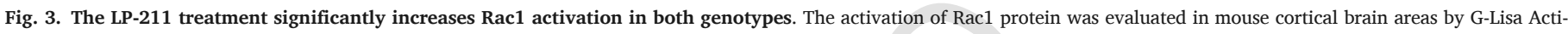

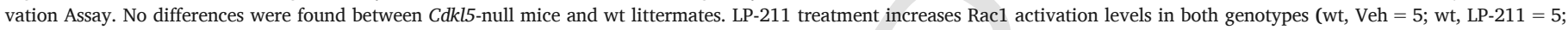
Cdkl5-null, Veh $=5$; Cdkl5-null, LP-211 = 4). Data are mean \pm SEM. Statistical significance was calculated by two-way ANOVA.

A

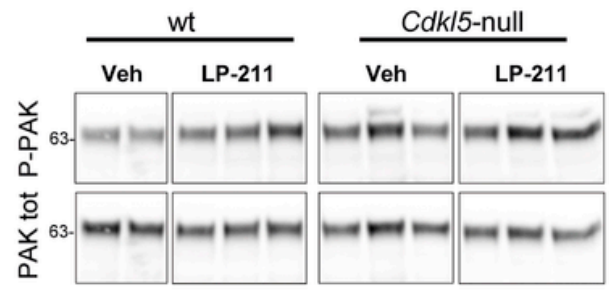

B Treatment: $p=0.003$

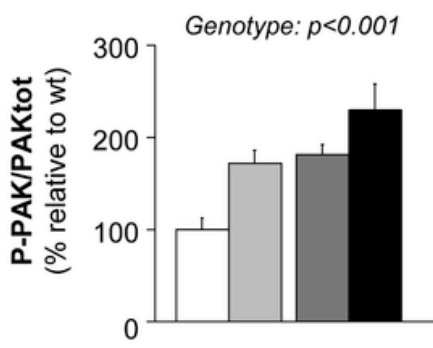

E

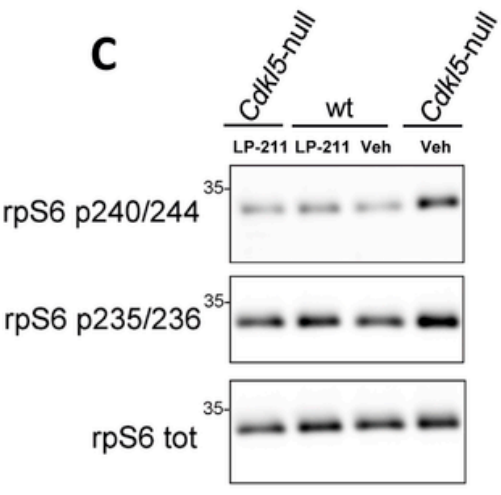

D

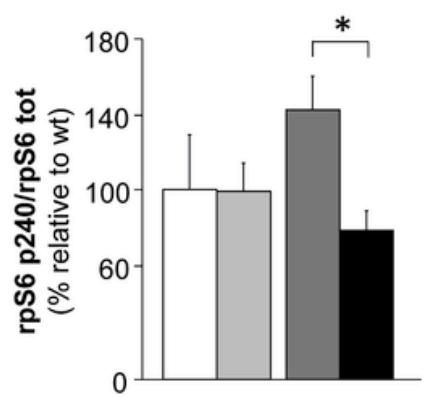

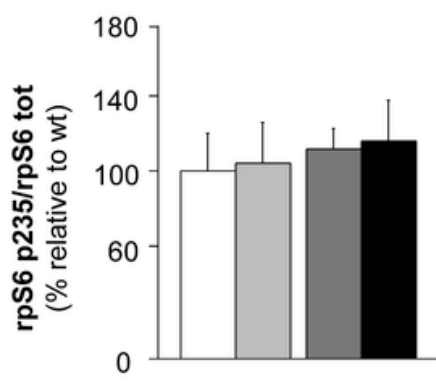

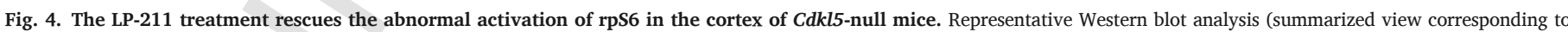

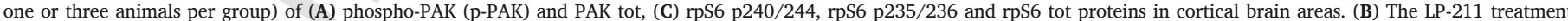

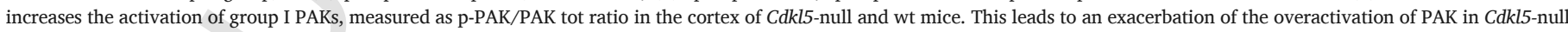

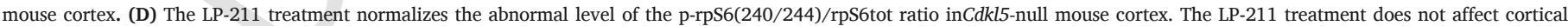

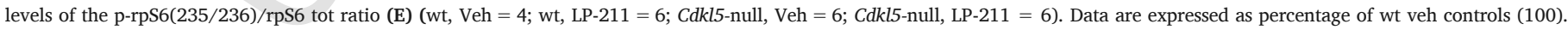
Data are mean \pm SEM. Statistical significance was calculated by two-way ANOVA. **p $<0,01 ; * \mathrm{p}<0,05$ after Tukey's post-hoc tests.

Cdkl5 levels. Interestingly, the LP-211 treatment slightly, but significantly increased Cdkl5 protein levels in the cortex of LP-211-treated wt mice, in comparison to wt controls [Genotype*Treatment interaction: $\mathrm{F}$
$(1,18)=11.6, \mathrm{p}=0.003$; post-hoc: $\mathrm{p}<0.01$; wt, veh: $100 \pm 0.2$ and wt, LP-211: $140 \pm 0.2$ (\% relative to wt)]. As expected, Cdkl5 was not detected in the brain of mutant mice. 
$5-H T_{7} R$ levels. We also evaluated whether the levels of the $5-\mathrm{HT}_{7} \mathrm{R}$ differ in the brain of Cdkl5-null mice compared to wt controls and LP-211 effects thereon. No significant genotype or treatment effects were found in cortex (Fig. S4).

\subsection{Cdkl5-null mouse brain shows defective mitochondrial functionality that is rescued by the LP-211 treatment}

Based on recent evidence suggesting a role for $5-\mathrm{HT}_{7} \mathrm{R}$ and Rho GTPases in the regulation of brain mitochondrial functionality (De Filippis et al., 2015a, 2015b; Valenti et al., 2017), we analyzed mitochondrial functionality in Cdkl5-null mouse brains.

Activity of Mitochondrial Respiratory Chain (MRC) complexes. We found reduced activity of the MRC complexes III, IV and V in Cdkl5-null mouse brains compared to wt controls [Fig. 5A; Repeated measure*Genotype*Treatment interaction: $F(4,32)=13.4 ; \mathrm{p}<0.001$; post-hoc: $\mathrm{p}<0.01]$. No difference was found in the activity of complexes I and II (Fig. 5A). A complete restoration in the activity of the defective MRC complexes in LP-211-treated Cdkl5-null mice was found [Fig. 5A; Repeated measure* Genotype*Treatment interaction; post-hoc: $\mathrm{p}<0.01$ compared to vehicle-treated Cdkl5-null mice for complexes IV and $\mathrm{V}$ and $\mathrm{p}<0.05$ compared to vehicle-treated Cdkl5-null mice for complex III].

Brain energy status evaluation. To evaluate if normalization of the activity of MRC complexes was associated with a normalization of their bioenergetic efficiency, the ATP production rate and ATP whole brain levels were measured (Fig. $5 \mathrm{~B}, \mathrm{C}$ ). In line with the results on complexes activity, Cdkl5-null mouse mitochondria showed a significant reduction in mitochondrial ATP production rate when supplied with the substrate for complex IV (ascorbate/TMDP), as energy source [Fig. 5B; Repeated measure* Genotype*Treatment interaction: $\mathrm{F}$ $(2,16)=3.2 ; \mathrm{p}=0.066 ;$ post-hoc: $\mathrm{p}<0.05]$. No changes were found when substrates for complexes I and II were used (Fig. 5B). Importantly, whole brain ATP levels were also reduced in Cdkl5-null mouse brain in comparison to wt controls [Fig. 5C; Genotype*Treatment interaction: $\mathrm{F}(1,12)=20.2 ; \mathrm{p}<0.001 ;$ post-hoc: $\mathrm{p}<0.01]$. LP-211 treatment completely rescued the defective mitochondrial ATP production and the reduced brain ATP levels in Cdkl5-null mice [Fig. 5B; ATP production: Repeated measure*Genotype*Treatment interaction; post-hoc:

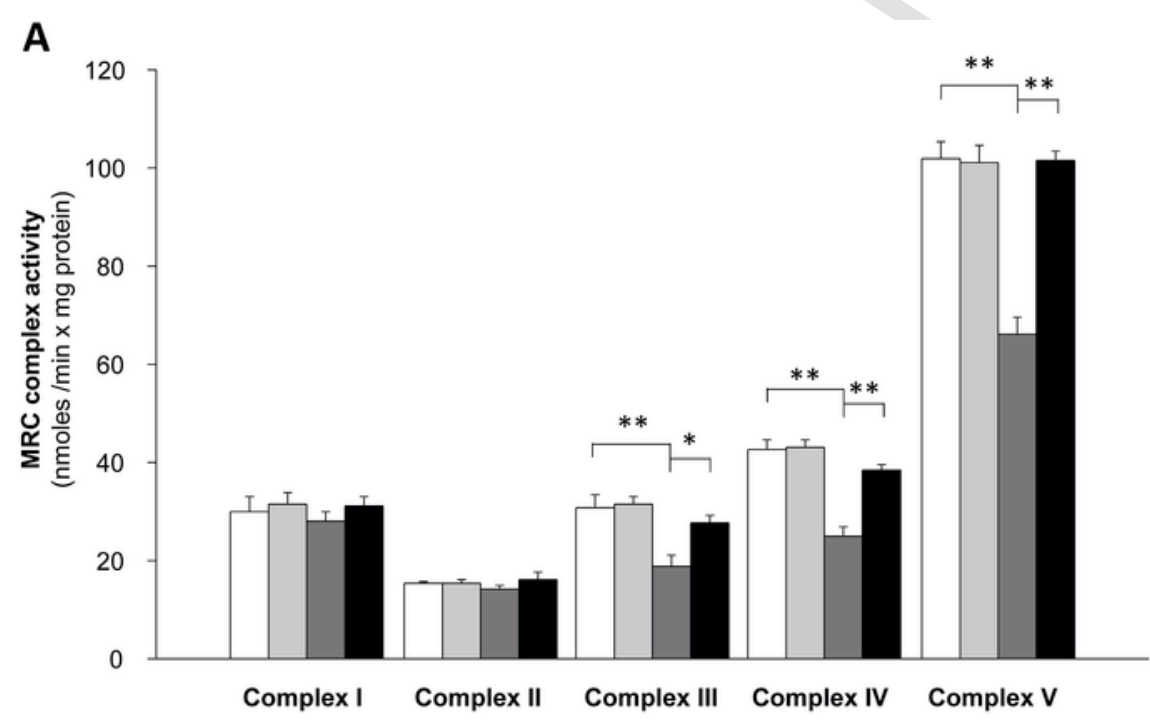

B

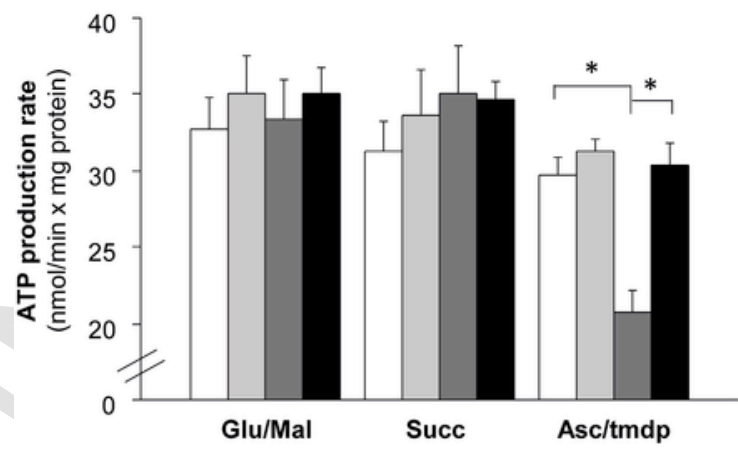

C

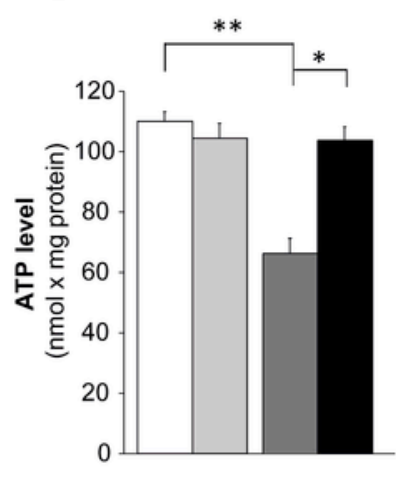

wt, veh

wt, LP-211

Cdkl5-null, veh

Cdkl5-null, LP-211

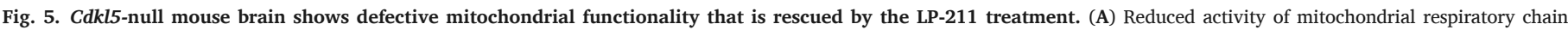

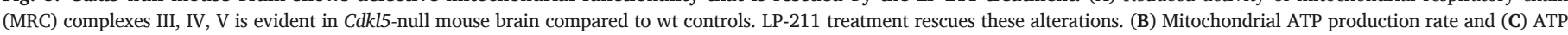

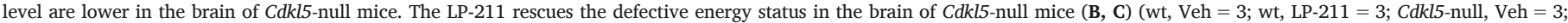
Cdkl5-null, LP-211 = 3). Data are mean \pm SEM. Statistical significance was calculated by two-way ANOVA. **p $<0,01 ;{ }^{*}$ p $<0,05$ after Tukey's post-hoc test. 
$\mathrm{p}<0.05$; Fig. 5C; whole brain ATP level: Genotype*Treatment interaction; post-hoc: $\mathrm{p}<0.01]$.

\section{Discussion}

This study provides the first characterization of the behavioural phenotype of Cdkl5-null mice at an advanced stage of the disease and demonstrates that 5- $\mathrm{HT}_{7} \mathrm{R}$ modulation, with the $5-\mathrm{HT}_{7} \mathrm{R}$ agonist LP-211, partially rescues the abnormal neurobehavioural phenotype of fully symptomatic Cdkl5-null male mice. In particular, in Cdkl5-null mice receiving the LP-211 treatment we found a normalization of PPI deficits and a complete restoration of rpS6 activation in cortical brain areas. Moreover, we demonstrate for the first time that mitochondria, the powerhouses of the cells, show important abnormalities at the functional level in Cdkl5-null brain and that such functional alterations can be persistently restored by modulation of brain $5-\mathrm{HT}_{7} \mathrm{R}$.

In spite of the progressive nature of $\mathrm{CDD}$, mouse studies have been so far focused on young animals (i.e. $2-4$ months of age) and no information is available on behavioural as well as brain molecular alterations in Cdkl5-null mice at an advanced stage of the disease. The present study provides the first comprehensive characterization of the behavioural phenotype displayed by 9-12-months old Cdkl5-null male mice. In particular, we found marked alterations in the general health status and in the locomotor profile, with Cdkl5-null mice showing an hypolocomotor profile in the home cage and hyperlocomotion in the open field, thus confirming previous data in young animals (Amendola et al., 2014; Jhang et al., 2017). An abnormal profile was also observed in the fear conditioning task, suggestive of a profound cognitive impairment in fully symptomatic Cdkl5-null mice. We cannot however exclude that the hyperactive profile shown by Cdkl5-null mice when exposed to novel contexts may account for the reduced freezing behaviour in this cognitive task (Amendola et al., 2014; Jhang et al., 2017).

Furthermore, the comprehensive battery of behavioural tests we carried out allowed us to identify the presence of severe PPI deficits in fully symptomatic Cdkl5-null mice, a measure of sensorimotor gating of the startle reflex (Swerdlow et al., 2001) that is known to be affected in patients with several neuropsychiatric disorders including schizophrenia (Braff et al., 2001), and in rodent models (Schwabe and Krauss, 2017). As PPI can be easily assessed in patients (Braff et al., 2001), present results provide to the clinical setting an innovative, non-invasive tool to test the efficacy of potential treatments for CDD. Further studies are however needed to uncover the developmental course of this behavioural alteration as a reduction in PPI was previously reported in two-months old Cdkl5-null mice, that just missed statistical significance (Okuda et al., 2018).

Of note, the LP-211 treatment rescued this behavioural alteration in Cdkl5-null mice. A link between PPI deficits and abnormal serotonin signaling has been clearly established, with either an increase or a decrease in serotonin signaling leading to PPI disruption (Fletcher et al., 2001). Moreover, based on human studies demonstrating that $5-\mathrm{HT}_{7} \mathrm{R}$ mRNA is downregulated in the dorsolateral prefrontal cortex of schizophrenics (East et al., 2002), several works have addressed and demonstrated the involvement of $5-\mathrm{HT}_{7} \mathrm{R}$ in regulation of the PPI response in rodents (Pouzet et al., 2002b; Semenova et al., 2008). Our results similarly suggest that $5-\mathrm{HT}_{7} \mathrm{R}$ may be critically involved in serotonin-dependent regulation of the sensorimotor gating processing. Since we did not observe any change in the levels of the $5-\mathrm{HT}_{7} \mathrm{R}$ in Cdkl5-null mouse brain, our results suggest that stimulation of the $5-\mathrm{HT}_{7} \mathrm{R}$ might have indirectly rescued $5-\mathrm{HT}_{7} \mathrm{R}$-independent defects in Cdkl5-null mouse brain. Indeed, several serotonin receptors have been found to be involved in the regulation of PPI (Pouzet et al., 2002a; Mitchell and Neumaier, 2008; Pogorelov et al., 2017). Moreover, other neurotransmitter systems including glutamate and dopamine play a role in regulating sensorimotor gating (reviewed in (Geyer et al., 2001)).
Another important finding of the present study concerns the demonstration that Cdkl5-null mouse brains display impaired mitochondrial OXPHOS and a consequent decrease in brain energy status. We found reduced activity of the complexes III, IV, V and decreased ATP production and whole brain levels. How the lack of Cdkl5 produces such a mitochondrial dysfunction in mouse brain is not yet clear. Both transcriptional and post-translational mechanisms may be involved (De Filippis et al., 2015b). Of note, high levels of oxidative stress markers have been found in CDKL5 patients (Pecorelli et al., 2011), that have been proposed to be due to mitochondrial dysfunction (Pecorelli et al., 2015). We clearly demonstrate here the occurrence of multilevel dysfunctions of brain mitochondria in Cdkl5-null mice, thus providing support to this hypothesis.

Interestingly, reactivation of mitochondrial respiratory chain complexes in Cdkl5-null mouse brain by the LP-211 treatment rescued the defective brain energy status. Present results are in line with previous studies reporting the beneficial effect of the LP-211 treatment on brain mitochondrial function of two mouse models of RTT (Valenti et al., 2017). Taken together, these data strengthen the suggested link between $5-\mathrm{HT}_{7} \mathrm{R}$ and mitochondria in mouse brain and add relevant information to previous studies demonstrating a role for the serotonergic system in the regulation of mitochondria homeostasis (Chen et al., 2007; de Oliveira, 2016).

In the present study, we focused on RhoGTPases signaling, based on previous evidence suggesting that these pathways may be altered in CDD (Chen et al., 2010). Contrary to our expectation, we found normal activation levels of Rac1 in Cdkl5-null mouse cortex at the tested age. These results are in contrast with previous in vitro studies suggesting that defective Rac1 activation may play a role in CDD pathogenesis (Chen et al., 2010; Barbiero et al., 2017). Since no data on younger animals are currently available we cannot however exclude that such inconsistency may be due to the advanced age of the experimental mice. Indeed, a recent study aimed at evaluating Rac1 signaling in the brain of Fragile X mouse model has uncovered an age-dependent effect, with the observed Rac1 overactivation disappearing in older animals (Pyronneau et al., 2017).

Evidence that CDKL5 pathogenesis changes as the disease progresses is in fact provided by the increased activation of rpS6 (p 240/244) and the lack of genotype differences in Akt activation we found in Cdkl5-null mouse cortex at an advanced stage of the disease, which are in contrast with the previously reported reductions in younger animals (9-12 months of age vs postnatal day 27 and 60) (Amendola et al., 2014; Della Sala et al., 2016). Moreover, recent evidence demonstrated age-dependent efficacy of pharmacological treatment strategies in Cdkl5-null mice, with drugs exerting promising beneficial effects in two-month old Cdkl5-null mice losing their effectiveness at an advanced stage of the disease (Fuchs et al., 2018a). Altogether, these data highlight the need for studies aimed at evaluating the developmental progression of the disease and for innovative therapeutic strategies to be applied at an advanced stage of the disease, when previously efficacious therapies may lose their effectiveness.

We found that the LP-211 treatment normalized the unexpected overactivation of rpS6 in Cdkl5-null cortex, in addition to PPI deficits and mitochondrial dysfunction. Given that Rac1 and AkT activation were found to be normal and were not affected by the LP-211 treatment, present results suggest that different upstream molecules of rpS6 are altered in Cdkl5-null mouse brain at an advanced stage of the disease, that may account for the beneficial effects of the treatment under investigation (Bokoch, 2003; Biever et al., 2015). Indeed, the 5- $\mathrm{HT}_{7} \mathrm{R}$ activation is known to stimulate several signaling cascades (Speranza et al., 2013; Guseva et al., 2014). Interestingly, among them, PKA activation has been intriguingly linked to de-phosphorylation of rpS6 at Ser240/ 244 (Bonito-Oliva et al., 2013) and to regulation of PPI (Kelly et al., 2007). 
Besides the overactivation of rpS6, increased activation of group I PAKs was also evident in Cdkl5-null mouse brain, that was exacerbated by the LP-211 treatment. This family of proteins is crucially involved in several neuronal processes potentially relevant for CDD. In fact, group I PAKs play a crucial role in modulating ultrastructural neuronal morphology in vivo and in regulating activity-dependent actin dynamics, underlying synaptic plasticity (Hayashi-Takagi et al., 2010; De Filippis et al., 2014b; Duffney et al., 2015). Moreover, overactivation of the Rac/ Pak pathway affects fear memory (Das et al., 2017), social learning (Molosh et al., 2014) and synaptic plasticity (Hayashi et al., 2004, 2007; Martinez and Tejada-Simon, 2011). Taken together, these results highlight the overactivation of Group I PAKs as a potential innovative target for the treatment of CDD at an advanced stage of the disease. Group I PAKs inhibitors are in fact increasingly recognized as promising candidates for the treatment of Fragile X and schizophrenia (Dolan et al., 2013; Hayashi-Takagi et al., 2014).

In conclusion, the present study provides the first evidence that the LP-211 treatment partially rescues the abnormal neurobehavioural phenotype of clearly symptomatic Cdkl5-null male mice. Abnormal PPI and reduced brain energy status due to mitochondrial dysfunction were also uncovered, for the first time, in Cdkl5-null mice at an advanced stage of the disease, thus providing innovative endophenotypes for CDD. Moreover, we provide here the first in vivo evidence that Cdkl5 in mouse cortex is involved in regulation of group I PAKs, a family of proteins that are crucially involved in several neuronal processes potentially relevant for CDD. Altogether, the present data highlight innovative endophenotypes and druggable molecular targets for this devastating disorder.

\section{Funding}

This work was supported by a research grant to B.D.F. from the University of Pennsylvania Orphan Disease Center on behalf of LouLou Foundation.

\section{Disclosure/conflicts of interest}

None of the authors declare financial interests or potential conflict of interests.

\section{Acknowledgements}

The authors are grateful to Maria Cristina Talamo and Vanessa Medici for technical assistance, Luigia Cancemi for animal care, Nadia Francia and Stella Falsini for administrative assistance.

\section{Appendix A. Supplementary data}

Supplementary data to this article can be found online at https:// doi.org/10.1016/j.neuropharm.2018.10.018.

\section{Uncited references}

De Filippis et al., 2015c; De Filippis et al., 2015d

\section{References}

Amendola, E., Zhan, Y., Mattucci, C., Castroflorio, E., Calcagno, E., Fuchs, C., Lonetti, G., Silingardi, D., Vyssotski, A.L., Farley, D., Ciani, E., Pizzorusso, T., Giustetto, M., Gross, C.T., 2014. Mapping pathological phenotypes in a mouse model of CDKL5 disorder. Plos One 9, e91613.

Bahi-Buisson, N., Nectoux, J., Rosas-Vargas, H., Milh, M., Boddaert, N., Girard, B., Cances, C., Ville, D., Afenjar, A., Rio, M., Heron, D., N'Guyen Morel, M.A., Arzimanoglou, A., Philippe, C., Jonveaux, P., Chelly, J., Bienvenu, T., 2008. Key clinical features to identify girls with CDKL5 mutations. Brain 131, 2647-2661.

Barbiero, I., Peroni, D., Tramarin, M., Chandola, C., Rusconi, L., Landsberger, N., Kilstrup-Nielsen, C., 2017. The neurosteroid pregnenolone reverts microtubule derange- ment induced by the loss of a functional CDKL5-IQGAP1 complex. Hum. Mol. Genet. 26, 3520-3530.

Biever, A., Valjent, E., Puighermanal, E., 2015. Ribosomal protein S6 phosphorylation in the nervous system: from regulation to function. Front. Mol. Neurosci. 8, 75.

Bokoch, G.M., 2003. Biology of the p21-activated kinases. Annu. Rev. Biochem. 72, 743-781.

Bonito-Oliva, A., Pallottino, S., Bertran-Gonzalez, J., Girault, J.A., Valjent, E., Fisone, G., 2013. Haloperidol promotes mTORC1-dependent phosphorylation of ribosomal protein S6 via dopamine- and cAMP-regulated phosphoprotein of $32 \mathrm{kDa}$ and inhibition of protein phosphatase-1. Neuropharmacology 72, 197-203.

Braff, D.L., Geyer, M.A., Swerdlow, N.R., 2001. Human studies of prepulse inhibition of startle: normal subjects, patient groups, and pharmacological studies. Psychopharmacology 156, 234-258.

Chao, H.T., Chen, H., Samaco, R.C., Xue, M., Chahrour, M., Yoo, J., Neul, J.L., Gong, S., Lu, H.C., Heintz, N., Ekker, M., Rubenstein, J.L., Noebels, J.L., Rosenmund, C., Zoghbi, H.Y., 2010. Dysfunction in GABA signalling mediates autism-like stereotypies and Rett syndrome phenotypes. Nature 468, 263-269.

Chen, Q., Zhu, Y.C., Yu, J., Miao, S., Zheng, J., Xu, L., Zhou, Y., Li, D., Zhang, C., Tao, J., Xiong, Z.Q., 2010. CDKL5, a protein associated with rett syndrome, regulates neuronal morphogenesis via Rac1 signaling. J. Neurosci. 30, 12777-12786.

Chen, S., Owens, G.C., Crossin, K.L., Edelman, D.B., 2007. Serotonin stimulates mitochondrial transport in hippocampal neurons. Mol. Cell. Neurosci. 36, 472-483.

Cifariello, A., Pompili, A., Gasbarri, A., 2008. 5-HT(7) receptors in the modulation of cognitive processes. Behav. Brain Res. 195, 171-179.

Costa, L., Spatuzza, M., D'Antoni, S., Bonaccorso, C.M., Trovato, C., Musumeci, S.A., Leopoldo, M., Lacivita, E., Catania, M.V., Ciranna, L., 2012. Activation of 5-HT7 serotonin receptors reverses metabotropic glutamate receptor-mediated synaptic plasticity in wild-type and Fmr1 knockout mice, a model of Fragile X syndrome. Biol. Psychiatry 72, 924-933.

Das, A., Dines, M., Alapin, J.M., Lamprecht, R., 2017. Affecting long-term fear memory formation through optical control of Rac1 GTPase and PAK activity in lateral amygdala. Sci. Rep. 7, 13930.

De Filippis, B., Chiodi, V., Adriani, W., Lacivita, E., Mallozzi, C., Leopoldo, M., Domenici, M.R., Fuso, A., Laviola, G., 2015a. Long-lasting beneficial effects of central serotonin receptor 7 stimulation in female mice modeling Rett syndrome. Front. Behav. Neurosci. 9, 86.

De Filippis, B., Musto, M., Altabella, L., Romano, E., Canese, R., Laviola, G., 2015b. Deficient purposeful use of forepaws in female mice modelling rett syndrome. Neural Plast. 2015, 326184.

De Filippis, B., Nativio, P., Fabbri, A., Ricceri, L., Adriani, W., Lacivita, E., Leopoldo, M., Passarelli, F., Fuso, A., Laviola, G., 2014a. Pharmacological stimulation of the brain serotonin receptor 7 as a novel therapeutic approach for Rett syndrome. Neuropsychopharmacology 39, 2506-2518.

De Filippis, B., Ricceri, L., Fuso, A., Laviola, G., 2013. Neonatal exposure to low dose corticosterone persistently modulates hippocampal mineralocorticoid receptor expression and improves locomotor/exploratory behaviour in a mouse model of Rett syndrome. Neuropharmacology 68, 174-183.

De Filippis, B., Ricceri, L., Laviola, G., 2010. Early postnatal behavioral changes in the Mecp2-308 truncation mouse model of Rett syndrome. Gene Brain Behav. 9, 213-223.

De Filippis, B., Romano, E., Laviola, G., 2014b. Aberrant Rho GTPases signaling and cognitive dysfunction: in vivo evidence for a compelling molecular relationship. Neurosci. Biobehav. Rev. 46 (2), 285-301.

De Filippis, B., Valenti, D., Chiodi, V., Ferrante, A., de Bari, L., Fiorentini, C., Domenici, M.R., Ricceri, L., Vacca, R.A., Fabbri, A., Laviola, G., 2015c. Modulation of Rho GTPases rescues brain mitochondrial dysfunction, cognitive deficits and aberrant synaptic plasticity in female mice modeling Rett syndrome. Eur. Neuropsychopharmacol 25, 889-901.

De Filippis, B., Valenti, D., de Bari, L., De Rasmo, D., Musto, M., Fabbri, A., Ricceri, L., Fiorentini, C., Laviola, G., Vacca, R.A., 2015d. Mitochondrial free radical overproduction due to respiratory chain impairment in the brain of a mouse model of Rett syndrome: protective effect of CNF1. Free Radic. Biol. Med. 83, 167-177.

de Oliveira, M.R., 2016. Fluoxetine and the mitochondria: a review of the toxicological aspects. Toxicol. Lett. 258, 185-191.

Della Sala, G., Putignano, E., Chelini, G., Melani, R., Calcagno, E., Michele Ratto, G., Amendola, E., Gross, C.T., Giustetto, M., Pizzorusso, T., 2016. Dendritic spine instability in a mouse model of CDKL5 disorder is rescued by insulin-like growth factor 1 . Biol. Psychiatry 80, 302-311.

Dolan, B.M., Duron, S.G., Campbell, D.A., Vollrath, B., Shankaranarayana Rao, B.S., Ko, H.Y., Lin, G.G., Govindarajan, A., Choi, S.Y., Tonegawa, S., 2013. Rescue of fragile X syndrome phenotypes in Fmr1 KO mice by the small-molecule PAK inhibitor FRAX486. Proc. Natl. Acad. Sci. U. S. A. 110, 5671-5676.

Duffney, L.J., Zhong, P., Wei, J., Matas, E., Cheng, J., Qin, L., Ma, K., Dietz, D.M., Kajiwara, Y., Buxbaum, J.D., Yan, Z., 2015. Autism-like deficits in shank3-deficient mice are rescued by targeting actin regulators. Cell Rep. 11, 1400-1413.

East, S.Z., Burnet, P.W., Kerwin, R.W., Harrison, P.J., 2002. An RT-PCR study of 5-HT(6) and 5-HT(7) receptor mRNAs in the hippocampal formation and prefrontal cortex in schizophrenia. Schizophr. Res. 57, 15-26.

Elia, M., Falco, M., Ferri, R., Spalletta, A., Bottitta, M., Calabrese, G., Carotenuto, M., Musumeci, S.A., Lo Giudice, M., Fichera, M., 2008. CDKL5 mutations in boys with severe encephalopathy and early-onset intractable epilepsy. Neurology 71, 997-999.

Fehr, S., Downs, J., Ho, G., de Klerk, N., Forbes, D., Christodoulou, J., Williams, S., Leonard, H., 2016. Functional abilities in children and adults with the CDKL5 disorder. Am. J. Med. Genet. 170, 2860-2869.

Fehr, S., Wilson, M., Downs, J., Williams, S., Murgia, A., Sartori, S., Vecchi, M., Ho, G., Polli, R., Psoni, S., Bao, X., de Klerk, N., Leonard, H., Christodoulou, J., 2013. The CDKL5 disorder is an independent clinical entity associated with early-onset encephalopathy. Eur. J. Hum. Genet. 21, 266-273. 
Fletcher, P.J., Selhi, Z.F., Azampanah, A., Sills, T.L., 2001. Reduced brain serotonin activity disrupts prepulse inhibition of the acoustic startle reflex. Effects of 5,7-dihydroxytryptamine and p-chlorophenylalanine. Neuropsychopharmacology 24, 399-409.

Fuchs, C., Fustini, N., Trazzi, S., Gennaccaro, L., Rimondini, R., Ciani, E., 2018a. Treatment with the GSK3-beta inhibitor Tideglusib improves hippocampal development and memory performance in juvenile, but not adult, Cdk15 knockout mice. Eur. J. Neurosci. 47, 1054-1066.

Fuchs, C., Gennaccaro, L., Trazzi, S., Bastianini, S., Bettini, S., Martire, V.L., Ren, E., Medici, G., Zoccoli, G., Rimondini, R., Ciani, E., 2018b. Heterozygous CDKL5 knockout female mice are a valuable animal model for CDKL5 disorder. Neural Plast. 2018, 9726950.

Fuchs, C., Trazzi, S., Torricella, R., Viggiano, R., De Franceschi, M., Amendola, E., Gross, C., Calza, L., Bartesaghi, R., Ciani, E., 2014. Loss of CDKL5 impairs survival and dendritic growth of newborn neurons by altering AKT/GSK-3beta signaling. Neurobiol. Dis. 70, 53-68.

Geyer, M.A., Krebs-Thomson, K., Braff, D.L., Swerdlow, N.R., 2001. Pharmacological studies of prepulse inhibition models of sensorimotor gating deficits in schizophrenia: a decade in review. Psychopharmacology 156, 117-154.

Guseva, D., Wirth, A., Ponimaskin, E., 2014. Cellular mechanisms of the 5-HT7 receptor-mediated signaling. Front. Behav. Neurosci. 8, 306.

Guy, J., Gan, J., Selfridge, J., Cobb, S., Bird, A., 2007. Reversal of neurological defects in a mouse model of Rett syndrome. Science 315, 1143-1147.

Hayashi-Takagi, A., Araki, Y., Nakamura, M., Vollrath, B., Duron, S.G., Yan, Z., Kasai, H., Huganir, R.L., Campbell, D.A., Sawa, A., 2014. PAKs inhibitors ameliorate schizophrenia-associated dendritic spine deterioration in vitro and in vivo during late adolescence. Proc. Natl. Acad. Sci. U. S. A. 111, 6461-6466.

Hayashi-Takagi, A., Takaki, M., Graziane, N., Seshadri, S., Murdoch, H., Dunlop, A.J., Makino, Y., Seshadri, A.J., Ishizuka, K., Srivastava, D.P., Xie, Z., Baraban, J.M., Houslay, M.D., Tomoda, T., Brandon, N.J., Kamiya, A., Yan, Z., Penzes, P., Sawa, A., 2010. Disrupted-in-Schizophrenia 1 (DISC1) regulates spines of the glutamate synapse via Rac1. Nat. Neurosci. 13, 327-332.

Hayashi, M.L., Choi, S.Y., Rao, B.S., Jung, H.Y., Lee, H.K., Zhang, D., Chattarji, S., Kirkwood, A., Tonegawa, S., 2004. Altered cortical synaptic morphology and impaired memory consolidation in forebrain- specific dominant-negative PAK transgenic mice. Neuron 42, 773-787.

Hayashi, M.L., Rao, B.S., Seo, J.S., Choi, H.S., Dolan, B.M., Choi, S.Y., Chattarji, S., Tonegawa, S., 2007. Inhibition of p21-activated kinase rescues symptoms of fragile X syndrome in mice. Proc. Natl. Acad. Sci. U. S. A. 104, 11489-11494.

Hedlund, P.B., 2009. The 5-HT7 receptor and disorders of the nervous system: an overview. Psychopharmacology 206, 345-354.

Hedlund, P.B., Danielson, P.E., Thomas, E.A., Slanina, K., Carson, M.J., Sutcliffe, J.G., 2003. No hypothermic response to serotonin in 5-HT7 receptor knockout mice. Proc. Natl. Acad. Sci. U. S. A. 100, 1375-1380.

Jhang, C.L., Huang, T.N., Hsueh, Y.P., Liao, W., 2017. Mice lacking cyclin-dependent kinase-like 5 manifest autistic and ADHD-like behaviors. Hum. Mol. Genet. 26, 3922-3934.

Kalscheuer, V.M., Tao, J., Donnelly, A., Hollway, G., Schwinger, E., Kubart, S., Menzel, C., Hoeltzenbein, M., Tommerup, N., Eyre, H., Harbord, M., Haan, E., Sutherland, G.R., Ropers, H.H., Gecz, J., 2003. Disruption of the serine/threonine kinase 9 gene causes severe X-linked infantile spasms and mental retardation. Am. J. Hum. Genet. $72,1401-1411$.

Kelly, M.P., Isiegas, C., Cheung, Y.F., Tokarczyk, J., Yang, X., Esposito, M.F., Rapoport, D.A., Fabian, S.A., Siegel, S.J., Wand, G., Houslay, M.D., Kanes, S.J., Abel, T., 2007. Constitutive activation of Galphas within forebrain neurons causes deficits in sensorimotor gating because of PKA-dependent decreases in cAMP. Neuropsychopharmacology $32,577-588$.

Khan, H.A., 2003. Bioluminometric assay of ATP in mouse brain: determinant factors for enhanced test sensitivity. J. Biosci. 28, 379-382.

Kilstrup-Nielsen, C., Rusconi, L., La Montanara, P., Ciceri, D., Bergo, A., Bedogni, F., Landsberger, N., 2012. What we know and would like to know about CDKL5 and its involvement in epileptic encephalopathy. Neural Plast. 2012, 728267.

Leopoldo, M., Lacivita, E., De Giorgio, P., Fracasso, C., Guzzetti, S., Caccia, S., Contino, M., Colabufo, N.A., Berardi, F., Perrone, R., 2008. Structural modifications of $\mathrm{N}-(1,2,3,4$-tetrahydronaphthalen-1-yl)-4-aryl-1-piperazinehexanamides: influence on lipophilicity and 5-HT7 receptor activity. Part III. J. Med. Chem. 51, 5813-5822.

Macri, S., Ceci, C., Onori, M.P., Invernizzi, R.W., Bartolini, E., Altabella, L., Canese, R., Imperi, M., Orefici, G., Creti, R., Margarit, I., Magliozzi, R., Laviola, G., 2015. Mice repeatedly exposed to Group-A beta-Haemolytic Streptococcus show perseverative behaviors, impaired sensorimotor gating, and immune activation in rostral diencephalon. Sci. Rep. 5, 13257.

Manente, A.G., Valenti, D., Pinton, G., Jithesh, P.V., Daga, A., Rossi, L., Gray, S.G., O'Byrne, K.J., Fennell, D.A., Vacca, R.A., Nilsson, S., Mutti, L., Moro, L., 2013. Estrogen receptor beta activation impairs mitochondrial oxidative metabolism and affects malignant mesothelioma cell growth in vitro and in vivo. Oncogenesis 2, e72.

Martinez, L.A., Tejada-Simon, M.V., 2011. Pharmacological inactivation of the small GTPase Rac1 impairs long-term plasticity in the mouse hippocampus. Neuropharmacology 61, 305-312.

Matthys, A., Haegeman, G., Van Craenenbroeck, K., Vanhoenacker, P., 2011. Role of the 5-HT7 receptor in the central nervous system: from current status to future perspectives. Mol. Neurobiol. 43, 228-253.

Mitchell, E.S., Neumaier, J.F., 2008. 5-HT6 receptor antagonist reversal of emotional learning and prepulse inhibition deficits induced by apomorphine or scopolamine. Pharmacol. Biochem. Behav. 88, 291-298.

Molosh, A.I., Johnson, P.L., Spence, J.P., Arendt, D., Federici, L.M., Bernabe, C., Janasik, S.P., Segu, Z.M., Khanna, R., Goswami, C., Zhu, W., Park, S.J., Li, L., Mechref, Y.S., Clapp, D.W., Shekhar, A., 2014. Social learning and amygdala disruptions in Nf1 mice are rescued by blocking p21-activated kinase. Nat. Neurosci. 17, 1583-1590.

Okuda, K., Takao, K., Watanabe, A., Miyakawa, T., Mizuguchi, M., Tanaka, T., 2018. Comprehensive behavioral analysis of the Cdkl5 knockout mice revealed significant enhancement in anxiety- and fear-related behaviors and impairment in both acquisition and long-term retention of spatial reference memory. PloS One 13, e0196587.

Pecorelli, A., Belmonte, G., Meloni, I., Cervellati, F., Gardi, C., Sticozzi, C., De Felice, C., Signorini, C., Cortelazzo, A., Leoncini, S., Ciccoli, L., Renieri, A., Jay Forman, H., Hayek, J., Valacchi, G., 2015. Alteration of serum lipid profile, SRB1 loss, and impaired Nrf2 activation in CDKL5 disorder. Free Radic. Biol. Med. 86, 156-165.

Pecorelli, A., Ciccoli, L., Signorini, C., Leoncini, S., Giardini, A., D'Esposito, M., Filosa, S., Hayek, J., De Felice, C., Valacchi, G., 2011. Increased levels of 4HNE-protein plasma adducts in Rett syndrome. Clin. Biochem. 44, 368-371.

Pogorelov, V.M., Rodriguiz, R.M., Cheng, J., Huang, M., Schmerberg, C.M., Meltzer, H.Y., Roth, B.L., Kozikowski, A.P., Wetsel, W.C., 2017. 5-HT2C agonists modulate schizophrenia-like behaviors in mice. Neuropsychopharmacology 42, 2163-2177.

Pouzet, B., Didriksen, M., Arnt, J., 2002a. Effects of the 5-HT(6) receptor antagonist, SB-271046, in animal models for schizophrenia. Pharmacol. Biochem. Behav. 71, 635-643.

Pouzet, B., Didriksen, M., Arnt, J., 2002b. Effects of the 5-HT(7) receptor antagonist SB-258741 in animal models for schizophrenia. Pharmacol. Biochem. Behav. 71, 655-665.

Pyronneau, A., He, Q., Hwang, J.Y., Porch, M., Contractor, A., Zukin, R.S., 2017. Aberrant Rac1-cofilin signaling mediates defects in dendritic spines, synaptic function, and sensory perception in fragile X syndrome. Sci. Signal. 10.

Ricceri, L., De Filippis, B., Fuso, A., Laviola, G., 2011. Cholinergic hypofunction in MeCP2-308 mice: beneficial neurobehavioural effects of neonatal choline supplementation. Behav. Brain Res. 221, 623-629.

Ricciardi, S., Boggio, E.M., Grosso, S., Lonetti, G., Forlani, G., Stefanelli, G., Calcagno, E., Morello, N., Landsberger, N., Biffo, S., Pizzorusso, T., Giustetto, M., Broccoli, V., 2011. Reduced AKT/mTOR signaling and protein synthesis dysregulation in a Rett syndrome animal model. Hum. Mol. Genet. 20, 1182-1196.

Ricciardi, S., Ungaro, F., Hambrock, M., Rademacher, N., Stefanelli, G., Brambilla, D., Sessa, A., Magagnotti, C., Bachi, A., Giarda, E., Verpelli, C., Kilstrup-Nielsen, C., Sala, C., Kalscheuer, V.M., Broccoli, V., 2012. CDKL5 ensures excitatory synapse stability by reinforcing NGL-1-PSD95 interaction in the postsynaptic compartment and is impaired in patient iPSC-derived neurons. Nat. Cell Biol. 14, 911-923.

Rusconi, L., Kilstrup-Nielsen, C., Landsberger, N., 2011. Extrasynaptic N-methyl-D-aspartate (NMDA) receptor stimulation induces cytoplasmic translocation of the CDKL5 kinase and its proteasomal degradation. J. Biol. Chem. 286, 36550-36558.

Rusconi, L., Salvatoni, L., Giudici, L., Bertani, I., Kilstrup-Nielsen, C., Broccoli, V., Landsberger, N., 2008. CDKL5 expression is modulated during neuronal development and its subcellular distribution is tightly regulated by the C-terminal tail. J. Biol. Chem. 283, 30101-30111.

Schwabe, K., Krauss, J.K., 2017. What rodent models of deep brain stimulation can teach us about the neural circuit regulation of prepulse inhibition in neuropsychiatric disorders. Schizophr. Res.

Semenova, S., Geyer, M.A., Sutcliffe, J.G., Markou, A., Hedlund, P.B., 2008. Inactivation of the 5-HT(7) receptor partially blocks phencyclidine-induced disruption of prepulse inhibition. Biol. Psychiatry 63, 98-105.

Speranza, L., Chambery, A., Di Domenico, M., Crispino, M., Severino, V., Volpicelli, F, Leopoldo, M., Bellenchi, G.C., di Porzio, U., Perrone-Capano, C., 2013. The serotonin receptor 7 promotes neurite outgrowth via ERK and Cdk5 signaling pathways. Neuropharmacology 67, 155-167.

Speranza, L., Labus, J., Volpicelli, F., Guseva, D., Lacivita, E., Leopoldo, M., Bellenchi, G.C., di Porzio, U., Bijata, M., Perrone-Capano, C., Ponimaskin, E., 2017. Serotonin 5-HT7 receptor increases the density of dendritic spines and facilitates synaptogenesis in forebrain neurons. J. Neurochem. 141, 647-661.

Swerdlow, N.R., Geyer, M.A., Braff, D.L., 2001. Neural circuit regulation of prepulse inhibition of startle in the rat: current knowledge and future challenges. Psychopharmacology 156, 194-215.

Valenti, D., de Bari, L., De Filippis, B., Henrion-Caude, A., Vacca, R.A., 2014. Mitochondrial dysfunction as a central actor in intellectual disability-related diseases: an overview of Down syndrome, autism, Fragile X and Rett syndrome. Neurosci. Biobehav. Rev. 46 Pt 2, 202-217.

Valenti, D., de Bari, L., Vigli, D., Lacivita, E., Leopoldo, M., Laviola, G., Vacca, R.A., De Filippis, B., 2017. Stimulation of the brain serotonin receptor 7 rescues mitochondrial dysfunction in female mice from two models of Rett syndrome. Neuropharmacology 121, 79-88.

Valenti, D., Tullo, A., Caratozzolo, M.F., Merafina, R.S., Scartezzini, P., Marra, E., Vacca, R.A., 2010. Impairment of F1F0-ATPase, adenine nucleotide translocator and adenylate kinase causes mitochondrial energy deficit in human skin fibroblasts with chromosome 21 trisomy. Biochem. J. 431, 299-310.

Wang, I.T., Allen, M., Goffin, D., Zhu, X., Fairless, A.H., Brodkin, E.S., Siegel, S.J., Marsh, E.D., Blendy, J.A., Zhou, Z., 2012. Loss of CDKL5 disrupts kinome profile and event-related potentials leading to autistic-like phenotypes in mice. Proc. Natl. Acad. Sci. U. S. A. $109,21516-21521$.

Wilcox, R.R., 1987. New designs in analysis of variance. Annu. Rev. Psychol. 38, 29-60.

Zhou, A., Han, S., Zhou, Z.J., 2017. Molecular and genetic insights into an infantile epileptic encephalopathy - CDKL5 disorder. Front. Biol. 12, 1-6.

\section{Glossary}

CDKL5 Deficiency Disorder : CDD

Cyclin-dependent kinase-like 5: CDKL5

Serotonin receptor $7: 5-\mathrm{HT}_{7} \mathrm{R}$

Intellectual disability : ID

Rett syndrome : RTT

Induced-pluripotent stem cells : iPSCs

Ribosomal protein : rp

Intraperitoneal : i.p.

Oxidative phosphorylation: OXPHOS

Wild-type: wt 
Prepulse inhibition : PPI

Mitochondrial Respiratory Chain : MRC

Dimethyl sulfoxide : DMSO

White noise: WN

$B L$ : baseline

CS : conditioned stimulus
US : unconditioned stimulus

Inter trial interval : ITI

Nomenclature

LP-211 : N-(4-cyanophenylmethyl)-4-(2-diphenyl)-1-piperazinehexanamide PubChem : CID:25107716 